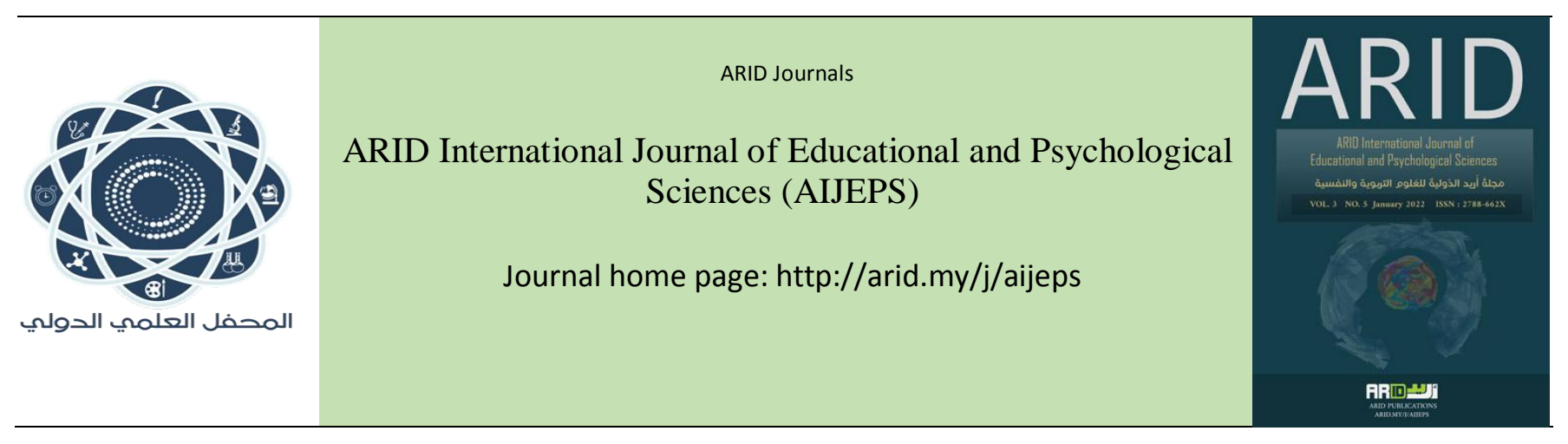

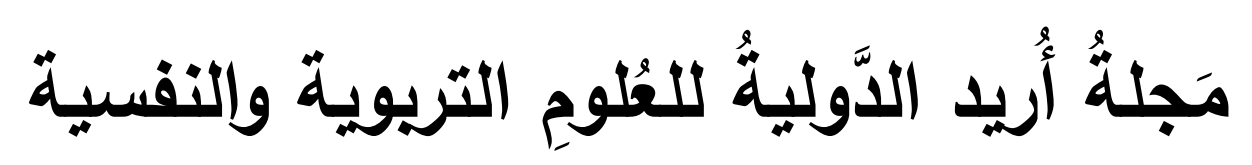

$$
\begin{aligned}
& \text { العدد } 5 \text { ، المجلد } 3 \text { ، كانون الثاني } 2022 \text { م }
\end{aligned}
$$

\title{
Attitudes of primary school teachers towards e-learning in teaching Arabic and the obstacles they face
}

$$
\begin{aligned}
& \text { اتجاهات معلمي المرحلة الأساسية نحو التعليم الإكتروني في تدريس اللغة العربية والمعوقات التي تواجههم }
\end{aligned}
$$

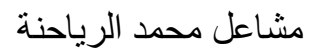

$$
\begin{aligned}
& \text { وزارة التربية و التعليم الأردنية } \\
& \text { * أنس عدنان عضيبات } \\
& \text { أكاديمية الأمبر الحسبن بن عبداله الثاني للحماية المدنية }
\end{aligned}
$$




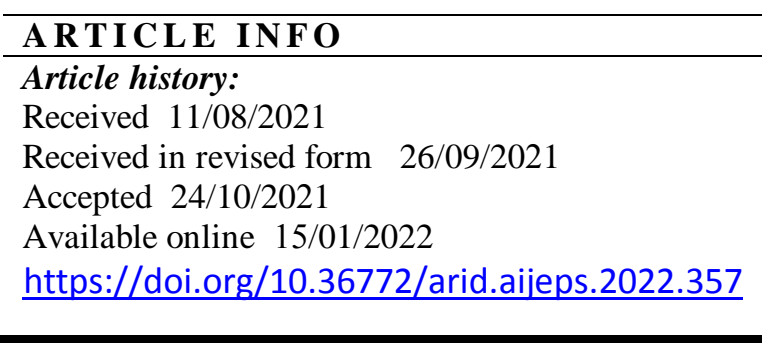

\begin{abstract}
The study aimed to identify the trends of primary stage laborers towards e-learning in teaching the Arabic language, and the obstacles they face from their point of view. The study relied on the descriptive and analytical approach, and the study population consisted of (170) male and female teachers working in public schools in Jerash governorate. The study sample (75) male and female teachers, who were chosen randomly. The results of the study showed that the attitudes of primary school teachers towards e-learning came at a positive level, with an arithmetic average (3.61), and the obstacles facing primary school teachers in e-learning came with a high degree, with an arithmetic average (3.84). The study recommended giving attention to providing electronic learning tools to include all schools, connecting all schools to the Internet and providing computers in proportion to the number of students in schools, and the study also recommended the necessity of dissolving all obstacles in e-learning, in terms of providing Internet labs in schools in proportion to the number of students and courses Scholastic.
\end{abstract}

Keywords: teacher attitudes, e-learning. 


\section{الملخص}

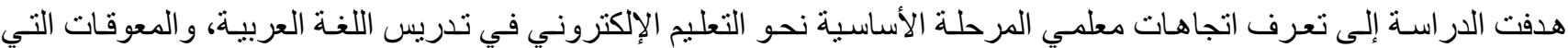

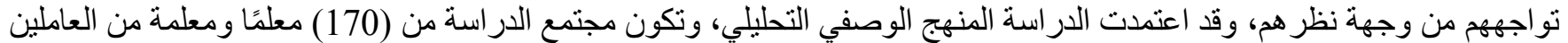

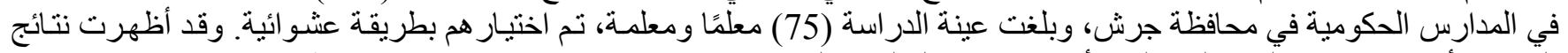

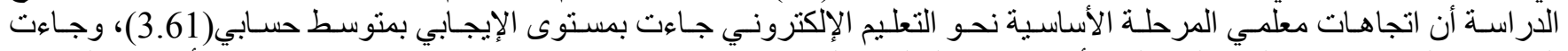

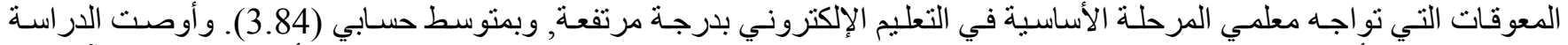

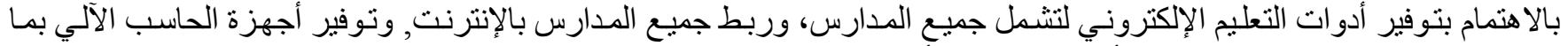

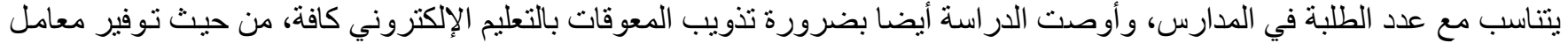

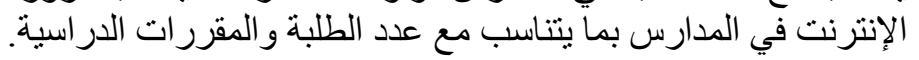

الكلمات المفتاحية: اتجاهات المعلمين، التعليم الإلكتروني. 
إن التطور ات المتسـار عة في عـالم التكنولوجيـا التي يشـهـها العصر الحسالي جعلتهـ يتصف بالانفجار التقني، وقد سـار عت مختلف المجالات و التخصصات إلى توظيف تلك المستحدثات التكنولوجية؛ لتطوير أدائها ومعالجـة مشكلاتها وتحسين مخرجاتها، و لا شك أن مجال التعليم هو من أبرز تلك المجالات؛ فقد ركزت التجارب والأبحاث والدراسات التربوية اهتمامهاــ منذ عشرات السنين وحتى الآنعلى توظيف التكنولوجيـا الحديثـة ومـا أنتجتـه من مستحدثات, وأجهزة, وأنظمـة, وبرمجيـات؛ لتحسين العمليـة التعليميـة في إجر اءاتها ومخرجاتها ومعالجة مشكلاتها. حيـث يعـد التعلـيم مـن أهـم المقومـات الأساسـية التـي ترتكـز عليهـا الـدول والحكومـات فـي بنـاء مسـتقبلها في عصـر المعلومـات و الإكترونيات؛ فمع ظهور أجهزة الحاسبات الشخصية وبر امجها التشغيلية إلى جانب تقنية المعلومات والاتصـالات والإنترنت وتطور هـا المستمر, ظهر التعلم الإلكتروني (E-Learning) و انتشر بشكل سريع، و أصبح من الو اضح أن له مستقبلا باهر اً، إلى حد أن المتوقع بل المؤكد أن التعليم الإلكتروني سيكون الأسلوب الأمثل والأكثر انتشار اً للتعليم و التدريب (السبيعي ومناصرة، 2017). و لأن مؤسسـات التعليم تعدد مؤسسـات اجتماعيـة تـؤدي دورًا رياديًا ومسؤولية كبرى في تحقيق تنميـة الر أسـمال الفكري, و التقدم التكنولوجي, وتتمية الاقتصاد المعرفي، والموازنة بين الحفاظ على الهويـة الذاتيـة والانفتاح على المجتمع العالمي، فإن التعليم في جميع أنحاء العالم المتقدم يشهد تغير ات وتحو لات؛ نتيجة للتطور ات التكنولوجيـة والمعلوماتيـة والحضسارية المعاصرة، وسبل توظيفها توظيفًا أمثل، ولعل هذا يمثل التحدي الحقيقي لمؤسسات التعليم في المجتمعات الأقل تقدمًا على وجـه الخصوص، و على العكس من ذلك أخذت المجتمعـات المتقدمة بأسباب التطور و العمل على مو اكبـة المستجدات الحديثـة بمـا يخدم مصـالحها، ويحقق أهدافها المنشودة (بيزان،

ويعد التعليم الإلكتروني بصوره المختلفة أهم وأبرز تللك البدائل؛ حيث بسهم هذا النوع من التعليم في تسـهيل الاتصـال بين المعلمين و الطلبة، والطلبة فيما بينهم، من خلال مجالس النقاش، والبريد الإلكتروني، وغرف الحوار الافتراضية؛ حيث يسهم التعليم الإلكتروني في بيان وجهات النظر المختلفة، والإحساس بالمساواة، وسهولة الوصول إلى المعلم، وإمكانية تحوير طريقة التدريس، وتوفير الدراسـة على مدار الوقت، والاستمرارية في الوصول إلى المنهاج، وسـهولة وتعدد طرق تقييم تطور المتعلمين، ومر اعـاة الفروق الفرديـة وتحويلـ التعليم إلى تعلم ذاتي (الحازمي، 2019).

فقد تغير دور المعلم والمتعلم بالتعليم الإلكترونـي بصورة واضحة، فلم يعد المعلم ملقنًا ولا المتعلم متلقيًا سلبيًا، فقد استلزم أن يكونـا نشطاء في أثناء الموقف التعليمي، وقد تأثرت المناهج ومحتواها وأنشطتها وطرق عرضها وأساليب تقويمها، كما أصبح إكساب المتعلمين مهار ات التعلم الذاتي، وأصبح الإتقان هو المعيار الأول لنظم التعليم وتكافؤ الفرص (البغدادي، 2015). 
ونظرًا لأهمية التعليم الإلكتروني من جانب، وقصور التعليم التقليدي في تحقيق أهدافه من جانب آخر، فإن التعليم الإلكتروني لا يمثل أحد الرو افد المهمة و المساندة للتعليم التقليدي فقط، بل الحل الأمثل لمقابلة التحديات التي تقابل التعليم التقليدي، وبالتالي لم يعد التعليم الإلكتروني خيارًا ثانويًا، بل إستر اتيجيًا يجب التعامل و التفاعل معه، وتسخيره لتحقيق نقلة نوعية في طريقة التعلم، ومن هنا جاءت هذه الدر اسة لتبين اتجاهات معلمي المرحلة الأساسية نحو التعليم الإلكتروني في تدريس المرحلة الأساسية والمعوقات التي تواجههم. مشكلة الدراسة: للمعلم في عصر الإلكترونيات وشبكات المعلومات والإنترنت دور كبير في إعداد طالب متعلم مؤهل ومدرب على مهار ات التعلم الذاتي, و البحث, و التحري, وكيفية التعامل مع المعلومات المتاحة, و اختبار ها, وتنظيمها، مما يسـهم في تتمية شخصيته بصورة متكاملة, وينمي ثقته بذاته, ويجعله فاعلًا في المجتمع قادرًا على التصدي لمواجهة مـا في مجتمعه من مشكلات؛ فقد اختلف دور المعلم في ظل التعليم الإلكتروني عن التعليم التقليدي، وتقييم ومعرفة اتجاهات التعليم الإلكتروني من أهم العو امل المسـاعدة على نجاحها وبخاصـة من قبل المعلمين، حيث ظهرت في الآونة الأخيرة عدة محاو لات لتطبيق التعليم الإلكتروني في مجال التعليم, لكن لم توجد محاو لات لتقييم ومعرفة اتجاهـات تلك التجارب من وجهة نظر معلمي اللغـة العربية، و المعوقات التي تو اجههم من وجهة نظر هم، وبخاصـة أن مـادة المرحلة الأساسية من المواد التي تجد نفورًا من الطلاب عند دراستها. وبناءً على ذلك, تتحدد مشكلة الدراسة الحالية في تعرف اتجاهات معلمي المرحلة الأساسية نحو التعليم الإلكتروني في تدريس المرحلة الأساسية والمعوقات التي تو اجههم, وذللك من خلال الإجابة عن الأسئلة التالية: أسئلة الار اسةة: 1. ما اتجاهات معلمي المرحلة الأساسية في مديرية تربية جرش نحو التعليم الإلكتروني من وجهة نظر هم؟ 2. ما المعوقات التي تو اجه معلمي المرحلة الأساسية في مديرية تربية جرش في استخدام التعليم الإلكتروني من وجهة نظرهم؟ أهمية الدراسة: تمتاز هذه الدر اسـة بأنها تبحـث موضـوعًا مهمًا، وهـو اتجاهـات معلمي المرحلـة الأساسية نحو التعليم الإلكترونسي في التـدريس, و المعوقات التي نو اجههح, ويؤمل أن تتمكن هذه الدر اسة من تحقيق أهدافها المرجوة, وأن تتمكن من: 1. إبراز أهمية التعليم الإلكتروني لما له من أهمية بالغة في تيسير تعليم المرحلة الأساسية بشكل عام؛ كونه من الإستر اتيجيات الحديثة في تدريس اللغة العربية. 2. معرفة اتجاهات معلمي المرحلة الأساسية نحو التعليم الإكتروني في تدريس المرحلة الأساسية, ومعرفة المعوقات التي تواجهم. 
1. تعرف اتجاهات معلمي المرحلة الأساسية في مديرية تربية جرش نحو التعليم الإلكتروني من وجهة نظر هم. 2. تعرف المعوقات التي تو اجه معلمي المرحلة الأساسية في مديرية تربية جرش في استخدام التعليم الإلكتروني من وجهة نظر هم. محددات الاراسة: المحدد المكاني: طُبقت هذه الدراسة في المدارس الحكومية التابعة لمديرية جرش. المحدد الزماني: تم إجر اء هذه الدراسة الميدانية خلال العام الدراسي 2020-2021م. المحدد البشري: جميع معلمي المرحلة الأساسية ومعلماتها في المدارس الحكومية التابعة لمديرية التربية والتعليم في محافظة جرش. المحدد الموضـوعي: تتاولت هذه الدراسـة اتجاهـات معلمي المرحلـة الأساسية نحو التعليم الإلكترونسي في تـريس المرحلـة الأساسية و المعوقات التي تواجههم. مصطلحات الاراسة: التعليم الإكتروني: منظومة تفاعلية ترتبط بالعملية التعليمية التعلمية، وتقوم هذه المنظومـة بالاعتمـاد على وجود بيئة إلكترونيـة رقميـة, تعرض للطالب المقرر ات والأنشطة بو اسطة الثبكات الإلكترونية والأجهزة الذكية(Berg, Simonson, 2018) التعليم الإكتروني إجرائيًا: مستوى ممارسة أثكال التعليم الإلكتروني المتمثلة في (الحاسوب التعليمي، الهاتف النقال، الإنترنت, و غير هـا من تقنيات و أدو ات التعليم الإلكتروني) لتوصيل المحتوى التعليمسي للمتعلمين مـن خـلال التو اصل بين المـتعلم والمعلم، وبين المتعلم و المحتوى التعليمي (محتوى مادة المرحلة الأساسية) بطريقة تفاعلية تمكنه من التعلم.

الاتجاهات: "حالة من الاستعداد العقلي والعصبي, تكونت نتيجة الخبرات و التجارب السـابقة التي يمر بها الإنسـان، وتعمل على توجيه الاستجابة نحو الموضو عات و المو اقف التي ترتبط به" (ملحم،2000 :33). المُعوّقات: ما يحدث في المدرسـة من الظروف و الإجر اءات الإداريـة أو الفنبـة, ممـا يمنـع أو يحدّ من استخدام المعلم لطر ائق التدريس الحديثة التي تعتمد على المشاركة الفاعلة من المتعلم، وتشجيعه على التفكير و التفاعل أثناء عملية التدريس، مثل طريقة التعلم التعاوني، وطريقة الاكتشاف الموجه، وطريقة الاستقصاء، وطريقة العصف الذهني، ونحوها, من الطر ائق والأساليب التي لا تعتمد على الإلقاء و التلقي السلبي من المتعلم (عبدالكريم، 396:2011). 
المعوقات التي تواجه المعلمين إجرائيًا: هي الدرجة الكلية التي يحصل عليها المفحوص من خلال إجابته عن فقرات المقياس المستخدم في الدراسة الحالية، وتتمنل في مجمو عة من العقبات التي تعوق معلمي المرحلة الأساسية من استخدام التعليم الإلكتروني. الإطار النظري والدراسات السابقة: يعد التعلم الإكتروني وسيلة فعالـة وهادفة ومهمة للحصول على المعرفة والاكتشـافات وقت حدوثها، وذلك لمو اكبة متغيرات هذا العصر و مسايرة مستجداته في الوقت ذاته، وقد أصبحت المجتمعات التي لا توظف وسائل و إمكانات وطر ائق التعلم الإكتروني مجتمعات غير متطورة, ويصعب عليها التعايش في هذا العصر المتلاطم بالأمواج المعلوماتية، ومن هذا المنطلق اهتم العالم بالتعليم الإلكتروني؛ لما له من أهمية و اضحة ومميز ات عديدة, و أصبح محل اهتمـام الحكومات و المؤسسـات العالميـة منها والإقليميـة، والدول المتقدمة التي حرصت على إدخال نظام التعلم الإكتروني في مؤسساتها التعليمية (البيطار ، 2016). ومنا هنا تبلور مفهوم التعلم الإلكتروني كإحدى صيغ التعلم الذاتي التي تركز على المتعلمين، وتعمل على إيصـال المعرفة لهم مهما كانت ظروفهم الاجتماعية والاقتصـادية، ومهما تكن مدة انقطاعهم عن التعليم النظسامي، والمسـافة الجغر افية بين مكان إقامتهم ومركز التعليم عن بعد، تحقيقا لمبدأ تكافؤ الفرص التعليمية وديمقر اطية التعليم (تمـام، 2010). على أن يعين لكل مجمو عة من المتعلمين معلم، يتولى إرشاد المتعلمين وتوجيههم، ويقدم لهم ما يحتاجون إليه من خدمات تتعلق بدر استهم، ويتولى متابعة المتعلمين أكاديميًا، ويتو اصل معهم عن طريق وسائل الاتصال المختلفة، ويعقد لقاءات بالمتعلمين بصورة منتظمة (بيتس، 2007). ويعد التعلم الإكتروني أحد النماذج التي تهتم بمساعدة المتعلم للحصول على المعرفة والعلم والتدريس الذي يحتاجها, وهو يعمل على توفير فرص التعليم ونقل المعرفة للمتعلمين ونطوير مهار اتهم على مختلف التخصصات, ولقد عرف صبري (2010) التعليم الإكتروني بأنه أحد أساليب التعلم الذاتي التي أفرزنها تكنولوجيا التعليم, و هو في أصله تعلم فردي, لكنه أدى إلى تعزيز نظام التعليم المفتوح ونظام التعليم المستمر. و عرفه مازن (2009) بأنه موقف تعليمي تعلمي, لوسـائل الاتصـال و التواصل بـه دور أساسي في التغلب على مشكلة المسافات التي تفصل بين المعلم والمتعلم, بحيث تتيح فرصة التفاعل المشترك بينهما. و عرفه سالم (2004) بأنه ذلك النوع أو النظام من التعليم الذي يقدم فرصًا تعليمية وتدريبية إلى المتعلم دون إثر اف مباشر من المعلم, ودون الالنز ام بوقت ومكان محددين لمن لم يستطع استكمال الدر اسـة أو يعوقه العمل عن الانتظـام في التعليم النظامي, ويعتبر بديلًا عن التعليم التقليدي أو مكملًا له، ويتم تحت إثر اف مؤسسة تعليمية مسؤولة عن إعداد المو اد التعليمية والأدوات اللازمة للتعلم الفردي, اعتمادًا على وسائط تكنولوجية عديدة, مثل الهاتف, و الر اديو, و الفاكس, و التلكس, و التلفزيون, و الكمبيوتر , والإنترنت, و الفيديو التفاعلي. ويقوم التعليم الإلكترونـي على مجموعـة مـن المبررات التـي أنثـار إليها (علي، 2012) في عدم التوازن في التوزيع الجغر افي للمؤسسات التعليمية؛ فهناك مناطق ودول تتوفر فيها مؤسسـات تعليمية, بينما تفتقر بعض المناطق و الدول لمؤسسـات التعليم, فالمناطق 
التي تفتقر لهذه المؤسسات يجد أبناؤ ها صعوبة في مو اصلة تعليمهح, ويأتي التعلم الإلكتروني ليحقق آمال وطموحات أبناء هذه المناطق, مـع ثورة وغزارة المعلومـات التي يشهـدها هذا القرن. لا شك أن ملامـح النصف الثناني من القرن الماضـي شهـ تدفقًا في المعلومـات وصسوبة متابعـة تدفقها بالنسبة لأي مؤسسة تعليميـة, ويعتمد هذا التدفق على التطور التكنولوجي السريع, و الزيادة الكبيرة في أعداد المتعلمين الر اغبين في التعليم. مما يجعل المؤسسات التعليمية التقليدية عاجزة عن توفير التعليم لتلك الأعداد المتز ايدة, في ظل الإمكانيات المحدودة للمؤسسات الحالية أمام عدد كبير من المتعلمين. كما يهدف التعلم الإلكتروني إلى تحقيق العديد من الأهداف التربويـة والعلميـة المهمهـ, كما يشير رونتري (1995)، ومازن (2009) و هي زيادة الفرص أمسام الأفر اد للتعليم المستمر، وتيسير إمكانية التعليم و إعادة التدريب، وتعويض الأفراد ممن فاتهم فرص التعليم، و إناحة الفرصة لهم للنمو المستمر وتحسين ظروف حياتهم، والمساعدة في عملية التقويم والتحديد الواضح لأهداف التقويم؛ حيث يتوقع أن تكون مهام التقويم مرتبطة بالقدرة على تحقيق الأهداف المنصوص عليها، الأمر الذي يمكن المتعلم من تحمل مسؤولية التعليم، وتقديم الخدمات التعليمية لمن فاتهم فرص التعليم أو من ير غبون في تغيير مهنتهج, مـع عدم ترك العمل أنثاء الدراسـة, وذلك بمر اعاة ظروف

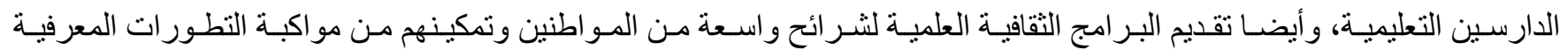
و التكنولوجية، والتوظيف الفعال لتكنولوجيا التعليم في العملية التعليمية لنشر الثقافة العلمية للمو اطن. و أيضا تحقيق مبدأ ديمقر اطية التعليم ودعم تكافؤ الفرص التعليمية وتطبيقاتها في أنواع التعليم ومسؤولياته المختلفة بما يتبعه للمحرومين من التعليم, من فرص الانخر اط فيه دون تمبيز فيما بينهم, لأسباب تتعلق بمكانتهم الاجتماعية أو الاقتصادية, أو بسبب العرق, أو الجنس, أو اللون, أو الدين، وأيضًا الإسهام في تطوير نوعية التعليح, باستتمار الوسائط التقنية الحديثة المتنوعة التي أثنتت فعالية المتعلم عوضًا عن التلقين السلبي السـائد، ومن أهم أهداف التعلم الإلكتروني بناء شخصية المتعلم بشكل إيجابي قادر على العطاء وحل المشكلات و التنمية الذاتية, وبالتنالي التنمية المجتمعيـة، AL- Muntashiri., والإسهام في حل المشكلات الناجمـة عن عجز مؤسسـات التعليم التقليديـة عن استيعاب الإعداد الهائلة المتز ايدة

وتحدد فوائد التعلم الإلكتروني في مجموعة من العو امل كما ذكر (إسماعيل، 2003) وهي: الملاءمة: حيث توفر الملاءمـة بين المعلم و المتعلم, و المرونة: يتتح للمتعلم خيار المشاركة حسب الرغبة, والتأثثر و الفاعلية: أثتتت البحوث التي أجريت على نظام التعلم الإكتروني أنه يوازي أو يفوق في التأثير و الفاعلية نظام التعليم التقليدي, وذلك عندما تستخدم هذه التقنيات بكفاءة. والمقدرة: الكثير من أنكال التعلم الإلكترونـي لا تكلف الكثير من المـال. و الإحسـاس المتعدد: هنـاك العديد من الخيارات في طرق توصيل المـادة الدراسية، منها المـادة الدر اسية المتلفزة, و التفاعل مع بر امج الكمبيوتر, و المادة الدراسية المسجلة في أشرطة كاسيت. 
و من أهم خصائص التعليم الإلكتروني أنها تساعد على تلبية الاحتياجات الاجتماعية والوظيفية والمهنية للملتحقين به؛ لما يتمتع بـه من مرونة وحداثة وتوفير للبدائل من جهة وارتباطاتهه بحاجات سوق العمل للعمالة المؤهلة من جهة أخرى، وينتفع هذا النمط من التعليم بالثورة التكنولوجية وثورة الاتصالات, ويعتمد بشكل رئيس على الوسـائط التكنولوجيـة الحديثة ووسائل الاتصـال المعاصرة، ويستجيب إلى عدد من مبادئ التعليم الإنساني الحديث مثل توفير الدافع للتعلم والمرونة في بيئة التعليم, وير اعي أساليب التعليم عند الأفراد وارتباط التعليم بحاجات الأفر اد الوظيفية والمهنية والثخصية والاجتماعية، ويعتمد على الطريقة النظاميـة المنهجية في تحديد البر امج الدراسية للطلبة, اعتمادًا على احتياجاتهم المهنية و الوظيفية, وبوفق طرق و أسـاليب وتقنيات متطورة في التعليم تتصف بالمرونـة، بالإضـافة إلى قدرته على اكتشاف أهداف المتعلم وقدر اته, وتحليلها وتفسير ها سو اء عند بدايـة التحاقه أو أثنـاء الدراسـة, وربط ذلك بـالبر امج التعليميـة، و تمكين المتعلم من المشاركة في برامج التعليم والتدريس دون فرض متطلبات تقليدية للالتحاق, ودون الحصول على درجة أكاديميـة أو أب شهادات أخرى، وقدرته على استيعاب أعداد متز ايدة من المتعلمين دون زيادة كبيرة في كلفة التعليم، و إمكان استخدام طرق تدريس ووسـائط تعليميـة متتوعـة، و إمكان استخدام التقويم والاختبـار ات باعتبار هـا أدو ات تشخيصية لتحليل مدى تحقيق أهداف التعليم (علي،

ويتطلب تتفيذ التعلم الإلكترونـي اتبـاع خطوات منظمـة, لخصـها مـازن (2010) في الآتي: يتم تصميم المسـاقات الدر اسية وإعداها و إنتاجها, سواء كانت مكتوبـة, أو مسموعة, أو مرئيسة, أو جميع هذه الوسـائل معاً إنتاجًا مركزيًا, من قبل أعضـاء الهيئة المشرفة على برنامج التعلم الإلكتروني, في ظل حاجات الفئات المستهدفة, ثم توزيع هذه المسـاقات ومـا ير افقها من برامج تعليمية في أوقات محددة لجميع المتعلمين, وتعريفهم بالبر امج التي تعتمد على أدوات الاتصـال العامـة كالإذاعة و التليفزيون, وأوقات بث هذه البر امج, وتعريف المتعلمين بممتلي المؤسسة التعليمية في مناطقهم, وطرق الاتصـال بهم, وتوفير مكان ارتباط مـع بعض الأجهزة والمو اد التعليمية التي تشكل بعض بر امج التعليم؛ وذلك لتقديم الاستشـار ات وتوزيع البرامج و المواد التعليمية, ثم وضـع نظام لتقويم تحصيل الطلاب وتقويم البر امج لتعديلها أو تطوير ها, و الاستغلال الأمثل لوسائط النقل المتاحة, و التركيز على العام منها كالإذاعة و التلفزيون, والاستعانة بنظام الحقائب التعليميـة في إعداد البر امج ودر استها؛ حيث إن هذا الأسلوب من الأسـاليب المتطورة ذات المردود الفعلي في التعلم الذاتي, ومقارنة الفروق الفردية لدى المتعلمين. وذكر هنداري (2009) أن التعليم الإكتروني يعتمد على ثلاثة مقومات أساسية هي: التكنولوجيا: حيث تمثل الدور المحوري في عملية التعلم الإلكتروني؛ فمفهوم البعد يفرض وجود رابط إلكتروني من الروابط التي توفر ها المستحدثات التكنولوجية مثل: الكمبيوتر، ووسائل الاتصال الحديثة، والإنترنت. و البر امج التطبيقية: من المعروف أن التعلم الإلكتروني يعتمد على التكنولوجيا, والتي تعتمد على مجموعة من البر امج التطبيقية كمعالجة النصوص، و الجداول، وقواعد البيانات، وبر امج التصفح في الإنترنت، وتصميم البرامج التعليمية، وتمثل 
البر امج التعليمية في هذا المجال العمود الفقري للتعليم عن بعد. والمحتوى العلمي: يصمم بطريقة خاصة؛ حيث ينت توزيع المحتوى على الإنترنت وو سائل الاتصال المختلفة, ويجب التركيز على لب موضوع الدراسة ونقاط اهتمام الدارسين. ومن أهم عيوب التعلم الإلكتروني: افتقار نظام التعلم الإكتروني لأسلوب التفاعل والاتصـال المباشر بين المعلم والطالب, وتغير المرونة التي يبديها التعلم الإكتروني نقطة ضعفه إذا ما قورنت بالتعليم التقليدي؛ لأنسه يقبل بدون معدل وبدون حضور, وقصور التعلم الإلكتروني على تدريس المو اد الإنسانية والاجتماعية ويفتقر إلى تدريس المواد الطبيعية(علي، 2012).

تناولت در اسة باليتا وشيرا ومبنج وليبي (Palitha, Chiara, Ming \& Libby, 2007) فاعلية التعليم الإلكتروني من خلال دمج البودكاست في مقرر المرحلة الأساسية والاتصالات لطلاب السنة الجامعية الأولى في جامعة (Kingston University), وتم إنشاء(6) بودكاست لتحسين أساليب التعلم ومهار اته, ومساعدة الطلاب على القيام بإعداد الأعمال الفصلية التي تُطلب منهم خلال فترة الدر اسة, وتم تقييم التجربة من خلال مجمو عتين الملاحظة ومقابلات شخصية للطلاب (6 طلاب) واستبانة طبقت على الطلاب (35 طالبًا). ووصف البحث سياقات للتعليم و التعلم, وكيفيـة إدخـال البودكاست كجزء مـن التعلم المدمج, وقيـاس مدى قدرتـه على تحقيق النتائج المرجوة, وتوصلت الدراسة إلى فاعلية التعليم الإلكتروني من خلال توظيف البودكاست في تفعيل عملية التعلم, ومساعدة الطلاب على أداء الأعمال الفصلية, وكانت اتجاهات الطلاب نحو التعلم إيجابية. وأجرى جامبو ومانكانو و هيلين (Helene, Mankato, Gumbo, 2012) دراسـة هدفت إلى تقيم أثر التدريب على التعليم الإكتروني للمعلمين في جنوب أفريقيا، حيث اتبعت الدراسة المنهج الوصفي على عينة من معلمين التربية و العلوم و الرياضيات عددهم (304) معلمين، وقد توصلت الدر اسـة إلى مجموعة من النتائج، أبرز هـا أن المعلمين استفادو ا بدرجة كبيرة، وأهميـة التدريب المستمر للمعلمين فبل الخدمة و أثنائها على التكنولوجيا الحديثة وتوظيفها في التربية و التعليم. أمـا در اسـة عبدالرحمن (2013) فهدفت إلى معرفة معوقات تطبيق التعليم الإلكتروني من وجهة نظر معلمي المرحلة الأساسية، وتكون مجتمـع الدر اسـة من معلمي مرحلـة التعليم الأساسي، وبلـغ عدد أفراد العينـة (90) معلمًا، واعتمدت الدراسـة المنهج الوصفي، و أشتارت أهم النتائج إلى عدم استعداد المعلمين لتطبيق التعليم الإلكتروني، وعدم توفر البنية التحتية، وعدم مو اكبة المنـاهج الحالية لتطبيق التعليم الإلكتروني بالمرحلة الأساسية. وسعت در اسـة أحمد (Ahmad, 2015) إلى تعرف أثر استخدام عقود التعلم الإلكتروني على الاستعداد للتعلم المنظم ذاتيا لدى طلاب المرحلة الأساسية, وتمثل مجتمع الدر اسة من جميع طلاب المرحلة الأساسية, وتم استخدام تصميم تجريبي يتضمن مجموعة و احدة من الطلاب, عددهم 36 طالبًا من طلاب الفرقة الثالثة بشعبة المرحلة الأساسية, وتم تقديم المحتوى بطريقة عقود التعلم الإلكترونية, وقد 
أسفرت نتائج الدر اسة عن فاعلية عقود التعلم الإلكتروني في تحسين الاستعداد للتعلم المنظم ذاتيا, و الذي انعكس على أداء الطلاب في مهار ات المرحلة الأساسية, وأوصى بالتوسع في توظيف عقود التعلم الإلكترونية في تدريس اللغة العربية. و أجرى شايم وأوفير (Chayim \& Offir, 2019) دراسة هدفت إلى اقتر اح تغيير في التعليم و التعلم الإلكتروني عن طريق تغيير دور المعلم, من خلال نموذج المعلم الوسيط، واتبعت الدر اسة المنهج التجريبي، وقسمت إلى مجموعتين مجموعة ضابطة تكونت من(6) معلمين, ومجمو عة تجريبية تكونت من(6) معلمين، وقد أظهرت نتائج الدراسة الإيجابية لصالح المجموعة التجريبية للمعلمين الذين تلقوا تدريبًا بو اسطة التعلم الإلكتروني غير المتزامن مع البيئة التي تتضمن محاضرات مسجلة بالفيديو. التعقيب على الدراسات السابقة:

بعد مر اجعة الدر اسات و البحوث السابقة, وجد الباحث أنها قدمت العديد من النتائج المهمة، ومن أبرز هذه النتائج: أنه يتوجب إنـراك الطلاب باستخدام تقنيات التعليم الإلكتروني؛ لأنها تعزز من مهار اتهم المختلفة، ويفترض توجيه الطلاب للمو اقع الإلكترونية التعليميـة على الشبكة العنكبوتيـة, و أيضًا وجوب إرسـال الواجبات على البريد الإلكترونسي, وتكليف الطلاب بتسليم الواجبـات عن طريق البريد الإكترونـي لتنميـة مههار ات استخدام تقنيـات التعليم الإلكترونـي، بالإضـافة إلى تدريب المعلمين و الطلبـة على استخدام تقنيـات التعليم الإلكتروني وممارستها. وتتفق هذه الدر اسة مع الدراسات السابقة في الموضوع الأساسي الذي تسعى لدراسته، و هو التعليم الإلكتروني، و استفادت هذه الدراسة من نتائج الدر اسات السابقة في إثر اء إطار ها النظري وتفسير نتائجها، ولعل ما يميز هذه الدراسة عن الدراسات السابقة، تتاولها فئسة مهــة في المجتمع، و هي فئة المعلمين. منهجية الاراسة: للإجابة عن أسئلة الدر اسة وتحقيق أهدافها، استخدم الباحث المنهج الوصفي التحليلي؛ لتناسبه مع طبيعة هذه الدراسـة، التي تهدف إلى الكشف عن اتجاهـات معلمي المرحلة الأساسية في المدارس الحكوميـة التابعة لمديريـة التربيـة والتعليم في محافظة جرش نحو التعليم الإكتروني في تدريس المرحلة الأساسية, و المعوقات التي تو اجههم من وجهة نظر هم. مجتمع الاراسة وعينتها: يتكون مجتمع الدر اسة من جميع معلمي المرحلة الأساسية التابعين لمديرية التربية والتعليم في المدارس الحكومية في محافظة جرش، لعام (2021/2020)، وبلغ عددهم (190) معلمًا ومعلمةً، وتكونت عينة الدر اسة من (75) معلمًا ومعلمة، تم اختيار هم بطريقة عثوائية، و الجدول رقم (1) يوضح تقسيم عينة الدراسة. 


\section{جدول(1): وصف خصائص عينة الاراسة}

\begin{tabular}{|c|c|c|c|}
\hline النسبة المئويـة & العدد & فئـة المتغير & المتغير \\
\hline 44 & 33 & ذكر & \multirow{2}{*}{ الجنس } \\
\hline 56 & 42 & أنثى & \\
\hline 49.33 & 37 & بكالوريوس & \multirow{3}{*}{ المؤهل العلمي } \\
\hline 30.67 & 23 & ماجستير & \\
\hline 20 & 15 & دكتور اه & \\
\hline 24 & 18 & أقل من 5 سنوات & \multirow{3}{*}{ الخبرة الوظيفية } \\
\hline 42.67 & 32 & 5- 10 سنوات & \\
\hline 33.33 & 25 & 11 سنوات فأكثر & \\
\hline 100 & 75 & & المجموع \\
\hline
\end{tabular}

أداة الدراسة:

تعددت أدوات البحث العلمي التي تستخدم في جمع المعلومات و البيانات، وبناءً على طبيعة البيانات التي يراد جمعها، و على المنهج المتبع في الدر اسـة، ظهر أن الأداة الأكثر ملاءمـة لتحقيق أهدافها، هي الاستبانة؛ إذ صمدت بعد مراجعة الأدبيـات، وأسـاليب البحث العلمي، و الدر اسات الميدانية ذات الصلة بموضوع الدر اسة. تكونت الأداة من (32) فقرة، تهتم بمعرفة اتجاهات معلمي المرحلة الأساسية نحو التعليم الإلكتروني في تدريس المرحلة الأساسية و المعوقات التي تو اجهم من وجهة نظر هم، وكانت الفقر ات من (1- 15) تهنم بمعرفة اتجاهات معلمي المرحلة الأساسية نحو التعليم الإلكتروني، وأمام كل فقرة خمسة بدائل، و هي: (دائمًا، غالبًا، أحيانًا، نادرًا، أبدًا)، و أعطيت الدرجات التالية بالترتيب (5، 4، 3، 2، 1) على التو الي، تم تقسيم المقياس إلى خمسة فئـات، و هي: (سلبي عـالٍ، وفئته من (1- 1.8)، سلبي وفئته (1.81-2.94)، محايد وفيتهـ (3.21-2.95)، إيجـابي و فئتسه (3.22-4.08)، و أخيـرًا إيجـابي عـالٍ وفئته (4.09-5))، في حبن كانـت الفقرات من (16-32) تقيس معوقات التعليم الإلكتروني من وجهة نظر معلمي المرحلة الأساسية, وأمسام كل فقرة خمسـة بدائل وهي: (مو افق بشدة وتعطى خمس درجات، دو افق وتعطى أربع درجات، محايد وتعطى ثنلاث درجات، غير مو افق وتعطى درجنان، غير موافق بشدة وتعطى درجـة و احدة)، وتم تقسيم المقياس إلى خمس فئات، و هي: (درجة ضعيفة جدًا وفئتها من (1- 1.8)، درجة ضعيفة وفئتها (1.81-3.09)، درجة متوسطة وفينتها (3.10-3.60)، درجة مرتفعة (4.61-4.07)، و أخير ا درجة مرتفعة جدًا وفئتها (4.08-5)). صدق أداة الاراسة: تم عرض الأداة على (8) محكمين من ذوي الخبرة والتخصص؛ لمعرفة آرائهم حول مدى انسجام الاستبانة ووضوحها وشموليتها، حيث شمل ذللك انتماء الفقر ات للمقياس ككل، وقد تم تعديل وصياغة الأسئلة بناءً على توصية المحكمين، وفي ضو ء مـا أبداه المحكمون من مقترحات للتعديل، تم القيام بإجر اء التعديلات التي اتفق عليها المحكمون، وفي ضوء ذلك تم تعديل وحذف عدد منها، بالإضـافة إلى إعادة صياغة بعض الفقرات لتشير بشكل مباشر ومختصر لما تهذف إليه الفقرة، مما حقق الصدق الظاهري لها. 
للتحقق من ثبات الاتساق الداخلي للأداة، تم حساب معامل ألفا كرونباخ (Cronbach,s Alpha)، على عينة استطلاعية مماثلة لعينة الدراسة مكونة من (15) معلمًا ومعلمة، وقد بلغت قيمة معامل الثبات للمقياس (810.) مـا يدل على ثبات عالٍ للاستبانة، وهي قيمة مناسبة لأغراض الدراسة. عرض النتائج ومناقشتها: النتائج المتعلقة بالإجابة عن السؤال الأول: "مـا اتجاهات معلمي المرحلة الأساسية في محافظة جرش نحو التعليم الإكتروني من

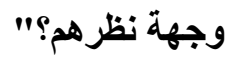
للإجابة عن هذا السؤ ال تم حساب المتوسطات الحسابية و الانحر افات المعيارية لاستجابات أفر اد العينة، و الجدول (2) يبين النتائج. جدول(2): الوسط الحسابي والانحراف المعياري لاستجابات أفراد العينة نحو التعليم الإكتروني

\begin{tabular}{|c|c|c|c|c|}
\hline المستوى & 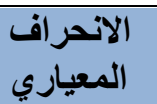 & المتوسطي المسابي & الفقرات & p \\
\hline محايد & 1.12 & 2.95 & على البريد الإلكتروني. على البريد الإلكترونـي وأكلف الطـلاب بتسليم الو اجبـات & 1 \\
\hline محايد & 1.05 & 2.98 & أكلف الطلاب بجمع الَّعلومات باستخدام الثبكة العنكبونية. & 2 \\
\hline محايد & 1.20 & 3.01 & أشتعربة. أن استخدام التعليم الإكترونـي يزيد من دافعيتي نحو تدريس اللغـة & 3 \\
\hline إيجابي & 1.11 & 3.22 & تعزيخ تدريس اللغة العربية. & 4 \\
\hline ايجابي & 1.28 & 3.81 & 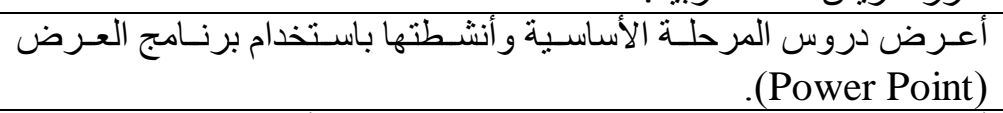 & 5 \\
\hline إيجابي & 0.99 & 4.08 & أُثنركة الطلاب باستخدام تقنيات التعليم الإلكتروني؛ لأنها تعزز من مهار اتهم & 6 \\
\hline إيجابي & 1.01 & 3.64 & 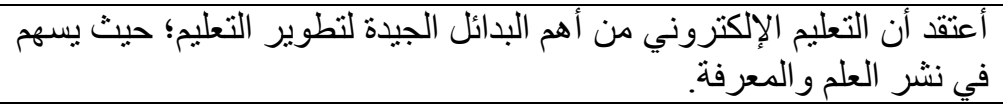 & 7 \\
\hline إيجابي & 0.99 & 3.42 & أستخدم التعليم الإلكتروني بشكل دائم ومنتظم. & 8 \\
\hline إيجابي & 1.03 & 4.02 & أقتلفت الطلاب بواجليم الإكتروني.ت علمية من قراءة كتب ومقالات ونشر ات من خلال & 9 \\
\hline إيجابي & 1.12 & 3.62 & أستخدم التعليم الإلكتروني بشكل مستمر في العملية التعليمية. & 10 \\
\hline إيجابي & 0.99 & 3.80 & أتيح وقتًَا كافيًا للمناقشة و العمل الجماعي من خلال تقنيات التعلم الإلكتروني. & 11 \\
\hline إيجابي & 1.05 & 3.99 & 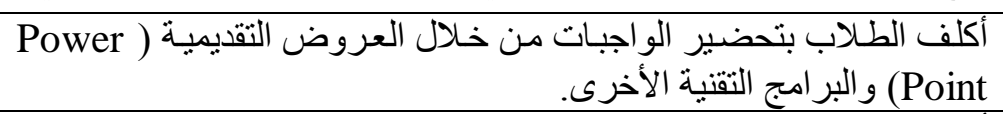 & 12 \\
\hline إيجابي & 1.09 & 3.85 & أتواصل مع المشرف التربوي إلكترونيًا من خلال تقنيات التعليم الإلكتروني. & 13 \\
\hline إيجابي & 1.10 & 4.03 & أوجه الطلاب للمو اقع الإلكترونية التعليمية على الثبكة العنكبوتية. & 14 \\
\hline إيجابي & 1.02 & 3.95 & الأستخدامي للطلابليم الإلكتروني يجعلني راضيًا عن توضيح دروس المرحلة & 15 \\
\hline إيجابي & & 3.61 & | الكلية & الدرج \\
\hline
\end{tabular}


يتبيّن من الجدول (2) أن المنوسطات الحسابية لإجابات أفر اد عينة الدراسة لاتجاهات المعلمين نحو التعليم الإلكتروني، نراوحت بين المستوى الإيجابي و المحايد، وبمتوسط حسابي تر اوح بين (2.95-4.08)، وجاءت الدرجة الكلية للأداة بمستوى الإيجابي، وبمتوسط حسابي (3.61)، حيث كان أعلاها للفقرة "أشرك الطلاب باستخدام تقنيات التعليم الإلكتروني لأنها تعزز من مهار اتهم المختلفة"، ثم تلاها "أوجه الطلاب للمو اقع الإلكترونية التعليمية على الثبكة العنكبوتيـة", في حين حصلت الفقرة "أرسل الو اجبات على البريد الإلكتروني و أكلف الطلاب بتسليم الو اجبات على البريد الإلكتروني" على أدنى متوسط حسابي (2.95)، وبانحر اف (1.12).

وتتفق نتائج الدر اسـة الحالية مـع در اسـة جامبو ومانكانو و هيلين (Helene, Mankato, Gumbo, 2012)؛ حيث إن المعلمين استفادوا بدرجة كبيرة من التعليم الإلكتروني، وأن لتدريب المعلمين على أدوات وتقنيات التعليم الإلكتروني أهمية كبيرة قبل و أثناء الخدمـة Palitha, Chiara, Ming \& Libby, ) على التكنولوجيا الحديثة وتوظيفها في التربية والتعليم، ودر اسـة باليتا وشير ا ومبنج وليبي 2007) التي توصلت إلى فاعلية التعليم الإكتروني من خلال توظيف البودكاست في تفعيل عملية التعلم ومسـاعدة الطلاب على أداء الأعمال الفصلية, وكانت اتجاهـات الطلاب نحو التعلم إيجابية, واتفقت أيضًا مـع در اسـة أحمد (Ahmad, 2015) و التتي أسفرت عن فاعلية عقود التعلم الإلكترونية في تحسين الاستعداد للتعلم المنظم ذاتبًا والذي انعكس على أداء الطلاب في مهارات المرحلة الأساسية, و أوصت بالتوسع في توظيف عقود التعلم الإلكترونية في تدريس اللغة العربية.

و أظهرت النتائج أن اتجاهات المعلمين نحو التعليم الإلكتروني جاءت بمستوى الإيجابي، ويعزو الباحث النتيجـة: إلى وعي المعلمين و المعلمات بمتطلبات تدريس المرحلة الأساسية باستخدام التعليم الإكتروني، وخبرتهم في هذا المجال لتسـيل عملية التعلم والتعليم، كمـا

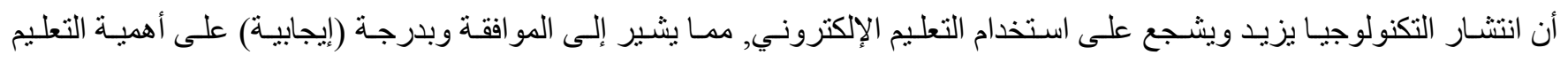
الإلكتروني في تدريس اللغة العربية.

النتائج المتعلقة بالإجابة عن السؤال الثاني: "ما المعوقات التي تواجه معلمي المرحلة الأساسية في محافظة جرش في استخدام التعليم الإلكتروني من وجهة نظر هم؟" للإجابة عن هذا السؤ ال تم حساب المنوسطات الحسابية والانحر افات المعيارية لاستجابات أفر اد العينة، و الجدول (3) يبين النتائج. 
جدول(3): الوسط الحسابي والاتحراف المعياري لاستجابات أفراد العينة للمعوقات التي تواجه المعلمين

\begin{tabular}{|c|c|c|c|c|}
\hline 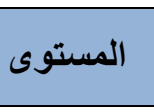 & الانحراف & المسبابي & 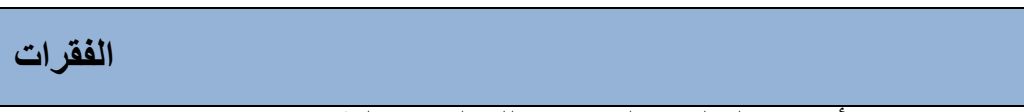 & م \\
\hline 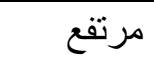 & 1.12 & 3.62 & عدم وضوح أهداف التعليم الإلكتروني للمعلمين و الطلاب. & 1 \\
\hline 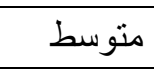 & 1.03 & 3.12 & ضعف المعلمين والطلبة في استخدام تقنيات التعليم الإلكتروني. & 2 \\
\hline مرتفع & 1.19 & 3.61 & ضعف التفاعل بين المعلمين و الطلبة بشكل مباثر . & 3 \\
\hline متوسط & 1.01 & 3.10 & ضعف التذريب و التأهيل للمعلمين والطلاب على تقنيات التعليم الإلكتروني. & 4 \\
\hline 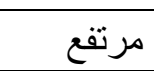 & 0.88 & 3.80 & زيادة التكلفة المادية من شر اء المعدات اللازمة والأجهزة. & 5 \\
\hline 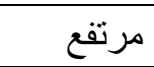 & 1.20 & 3.87 & صعوبة تنفيذ بعض الوسائل في التعليم الإلكتروني. & 6 \\
\hline 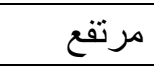 & 0.99 & 4.07 & تمسك المعلم بطرق التدريس التقليدية. & 7 \\
\hline مرتفع & 0.98 & 4.01 & الاعتقاد بأن استخدام التعليم الإلكتروني مضيعة للوقت. & 8 \\
\hline 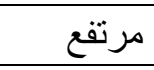 & 1.02 & 3.99 & قلة وجود أجهزة حاسب آلي عند الطلبة لاستخدام التعليم الإلكتروني. & 9 \\
\hline 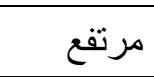 & 0.67 & 4.05 & زمن الحصة لا يكفي لاستخدام التعليم الإلكتروني في تدريس اللغة العربية. & 10 \\
\hline مرتفع جدًا & 0.75 & 4.16 & تدني فاعلية الأجهزة و التقنيات التعليمية المتوفرة بالمدارس. & 11 \\
\hline مرتفع & 1.21 & 3.85 & قلة تشجيع المشرف التربوي للمعلمين نحو الإفادة من التعليم الإلكتروني. & 12 \\
\hline مرتفع جدًا & 0.75 & 4.22 & ضعف شبكة الإنترنت عند استخدام التعليم الإلكتروني. & 13 \\
\hline مرتفع جدًا & 0.06 & 4.30 & 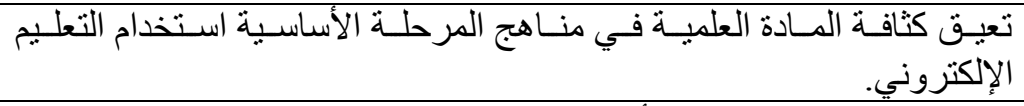 & 14 \\
\hline 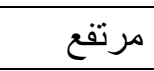 & 1.22 & 3.76 & قلة معرفة الطلاب باستخدام أنواع التعليم الإلكتروني. & 15 \\
\hline مرتفع & 1.04 & 3.84 & انخفاض دافعية الطلاب نحو استخدام التعليم الإلكتروني. & 16 \\
\hline مرتفع & 0.99 & 3.92 & تدني ملاءمة المادة التعليمية للتعلم الإلكتروني. & 17 \\
\hline 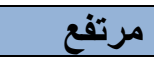 & & 3.84 & 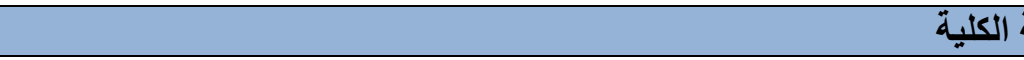 & 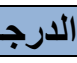 \\
\hline
\end{tabular}

يتبيّن من الجدول (3) أن المتوسطات الحسابية لإجابات أفر اد عينـة الدر اسـة للمعوقات التي تواجه معلمين اللغـة العربية، تر اوحت بين المستوى المرتفع و المرتفع جدًا، وبمنو سط حسابي تر اوح بين (3.10-4.30)، وجاءت الدرجة الكلية للأداة بمسنوى المرتفع، وبمنوسط حسابي (3.84)، حيث كان أعلاها للفقرة "تعيق كثافة المادة العلمية في منـاهج المرحلة الأساسية استخدام التعليم الإلكتروني"، ثم تلاهـا "ضعف شبكة الإنترنت عند استخدام التعليم الإلكتروني"، في حين حصلت الفقرة "ضعف التندريب والتأهيل للمعلمين و الطلاب على تقتيـات التعليم الإكترونـي"، على أدنى منوسط حسـابي (3.10)، وبـانحر اف (1.01). وتتفق الدراسـة الحاليـة مـع در اسـة عبدالرحمن (2013) و التي هدفت إلى معرفة معوقات تطبيق التعليم الإلكتروني من وجهة نظر معلمي المرحلة الأساسية، وأشـارت أهم النتائج إلى عدم استعداد المعلمين لتطبيق التعليم الإلكتروني، وعدم توفر البنيـة التحتيـة، وعدم مواكبـة المنـاهج الحاليـة لتطبيق التعليم الإكتروني بالمرحلة الأساسية. أظهرت النتائج أن المعوقات التي تو اجه معلمي المرحلة الأساسية في التعليم الإلكتروني من وجهة نظر هم جاءت بدرجـة مرتفعـة، ويعزو الباحث ذلك إلى عدم تدريب المعلمين بشكل كافٍ على استخدام تقنيات التعليم الإلكتروني, أو تدني قدرتهم على استخدام التعليم 
الإلكتروني, أو قلة و عيهم بأهمية ومميزات التعليم الإلكتروني، كما وقد تكون المقررات الدراسية سببًا في المعوقات التي لا يمكن تغطيتها بالتعليم الإلكتروني، فضـلً عن عدم تو افر أدوات التعليم الإلكتروني المساندة داخل المدارس، وضعف شبكة الإنترنت، لهذه الأسباب جاءت المعوقات بشكل مرتفع.

التوصيات:

في ضوء النتائج التي توصلت لها الدراسة، يوصي الباحث بما يأتي:

1. عقد دورات وورشات عمل للمعلمين والطلبة على حد سواء، تنمي اتجاهاتهم نحو التعليم الإلكتروني، وتدربهم على طرق استخدامه. 2. الاهتمام بتوفير أدوات التعليم الإكتروني لتشمل جميع المدارس، وربط جميع المدارس بالإنترنت وتوفير أجهزة الحاسب الآلي بمـا

$$
\text { يتناسب مع عدد الطلبة في المدارس. }
$$

3. تعديل مناهج المرحلة الأساسية ليتو افق تطبيقه مع التعليم الإلكتروني.

4. تذويب المعوقات بالتعليم الإلكتروني كافة، من حيث توفير معامل الإنترنت في المدارس وبما يتتاسب مـع عدد الطلبة والمقررات

الدراسية.

5. إجر اء المزيد من الدراسـات التربويـة حول الاتجاهـات و التعليم الإلكتروني, من حيث الأبعاد والتطبيقات في المؤسسـات التربويـة و غير ها من المؤسسات. 
إسماعيل، عفاف (2010). التعلم الإلكتروني في مجتمع المعرفة من منظور إسلامي. المؤتمر الدولي الثالث (دور التعلم الإكترونـي في تعزيز مجتمعات المعرفة). البحرين في الفترة من الإن 6 إلى فئى 8 أبريل.

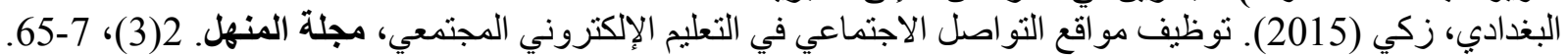

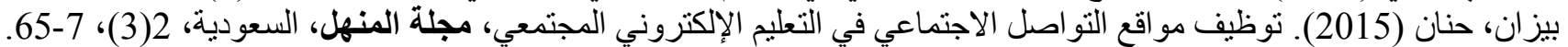
بيتس، دبيليو (2007). التكنولوجيا والتعليم الإلكتروني والتعليم عن بعد. مكتبة العبيكان، السعودية، التئية الرياض.

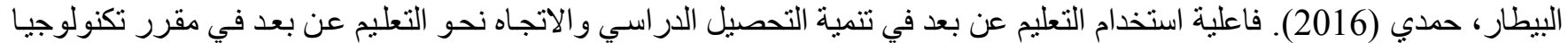

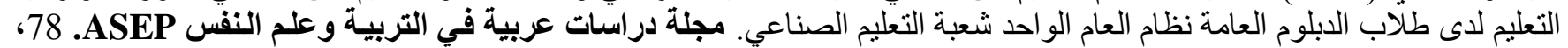
.39-17

تمام، شادية (2010). الجودة في برنامج التعليم المفتوح في ضوء الاتجاهات العالمية والاحتياجات المحلية. القاهرة.

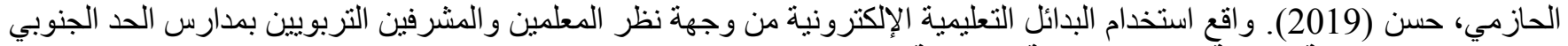

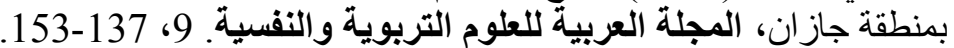

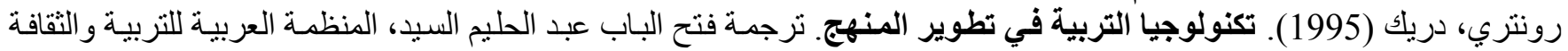
و العلوم المركز العربي للتقنيات التربوية، الكويت.

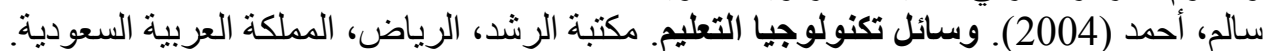

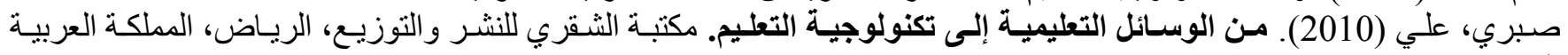
السعودية. السبيعي، عايض ومناصرة الطية، محمد (2017). و اقع استخدام التعلم الإلكتروني في تدريس مـادة المرحلة الأساسية بالمرحلة المتوسطة

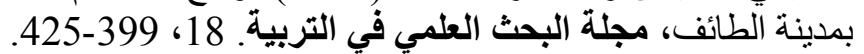

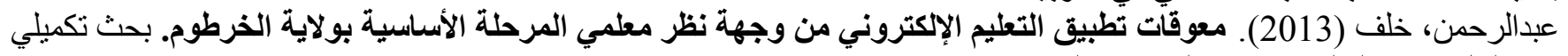
مقدم لنيل درجة الدبلوم، جامعة السودان، السوان السودان.

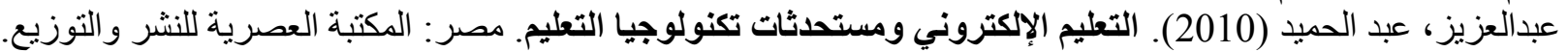

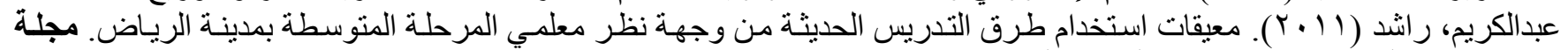

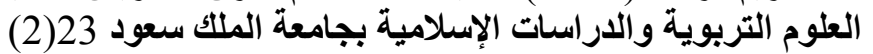

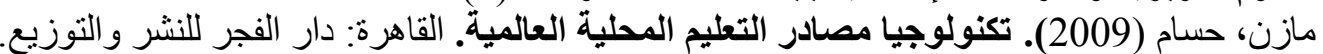

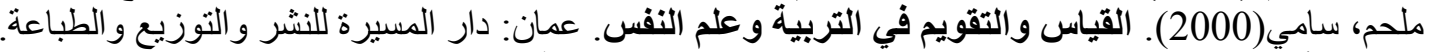

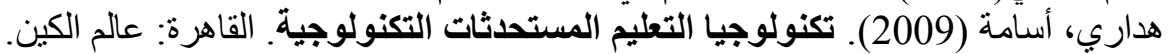

المراجع الأجنبية:

Chayim B.\&; Offir,A. (2019). Combine Asynchronous Distance Learning Via Videotaped Lecture. Journal of Educators Online. Model of the Mediating Teacher in Distance Learning Environments: Classes That ، A · 16(1).

Ahmad, S. (2015). The effect of using electronic learning contracts Elf student's self - directed learning readiness. Journal of psychological and educational research, faculty of education - Banham university.

Berg, G., Simonson, M. (2018). Distance learning. Britannica. https://www.britannica.com/topic/distance-learning

Gumbo, M. Mankato, M. \& Helene, M., (2012). The impact of Inservice technology training programmers on technology teachers, Journal of Technology Studies, 38(1), 23-33.

Mallareddy, K., (2010). Advantage and limitations of virtual classroom in Telegu language teaching. Iosr journal of humanities and social science, 15(1), 54-56.

Marie- Anne, V. (2010). Contextualization foreign language instruction: effective classroom strategies and blackboard based virtual learning. International journal of arts and science, 3(11), 116-122.

Palitha, E. Chiara, R. Ming, N. \& Libby, R. (2007). Podcasting to provide teaching and learning support for an undergraduate module on English language and communication, Turkish Online Journal of Distance Education - TOJDE, 8(3).

Al-Muntashiri , Ali Ahmed Abdullah.(2020). The technical Approach for Teaching Arabic , Arid International Journal of Educational and Psychological Sciences ,VOL.1 NO.2, July,(pp109-117). 